\title{
Characterization of high order modes of plasmonic antenna formed by nanoparticle/thin film hybrid structures
}

\author{
Shiuan-Yeh Chen ${ }^{\mathrm{a}, \mathrm{b}, \mathrm{c}}$, Yaroslav Urzhumov ${ }^{\mathrm{c}}$, David R. Smith ${ }^{\mathrm{c}}$, Anne A. Lazarides ${ }^{\mathrm{b}}$ \\ ${ }^{a}$ Dept. of Applied Materials and Optoelectronic Engineering, National Chi Nan University, \\ Puli, Taiwan, 54561 \\ ${ }^{b}$ Dept. of Mechanical and Materials Science, Duke University, Durham, NC, 27705 \\ ${ }^{\mathrm{c}}$ Dept. of Electrical and Computer Engineering, Duke University, Durham, NC 27705
}

\begin{abstract}
The plasmonic modes of a nano-antenna formed by a nanoparticle/thin film hybrid system are investigated. Plasmonic nano-antennas are well-known for their capabilities to concentrate electromagnetic wave into extreme small region and couple the emission from active materials in proximity to the antennas into far-field region. Previously, we have shown through direct measurement of emission profile images that the nano-antennas not only enhance Raman emission but also systematically direct inelastic emission to the far-field through the dipole mode. We also showed that high order modes of the hybrid structure can be detected. Here, the higher order plasmonic modes are further characterized through simulations of spectrum and scattering profile. Through spectral simulation with improved resolution, two distinct modes are found to contribute to the broad band high order mode. One has dipole-like behavior and the other has quadrupolelike behavior. The modes are characterized both through near-field distribution and far-field scattering profiles. The quadrupole-like mode can be excited by both p- and s-polarized light whereas the dipole-like mode is only excited by ppolarized light. These high order modes are not as bright as the dipole mode in the far-field spectrum but have substantial near field enhancement which can be utilized for surface-enhancing spectroscopy and sensing. In addition, characterization of high order modes may serve to clarify the interaction between nano-antenna and active materials and will lead to design rules for applications of active plasmonic structures in integrated optical circuits.
\end{abstract}

Keywords: nano-antenna, plasmonic modes, nanoparticles, thin film, near field, far field, scattering

\section{INTRODUCTION}

Plasmonic nanoparticles can be treated as optical nano-antennas. These nano-antennas can concentrate the far-field excitation into a small region even up to few $\mathrm{nm}^{2}$, forming hot spots. These hot spots strongly interact with molecules or active materials to enhance the scattering or absorption process, e.g. SERS (surface enhanced Raman scattering). Also, these nano-antennas can modify the far-field properties of the emission from the materials interacting with them.

The particle/film hybrid structure is a simple form of nano-antenna. This structure has been used for SERS and studied theoretically and experimentally. According to Nordlander's study,[1] in addition to dipole mode, high order modes could exist in this configuration. We have observed a spectral band to the blue of the primary gap dipole mode in single antenna spectra collected from gold particles on gold films.[2] From comparison with simulated spectra, the band appeared to correspond to a mode excited most efficiently by p-polarized incident light.

In this work, we report further characterization of scattering from this higher frequency band. Spectra collected under polarized illumination confirm that the dominant higher order $(\mathrm{HO})$ mode is excited by p-polarized radiation at a longer wavelength than the wavelength of the brightest mode observed under s-polarized illumination. Filtered images of scattering at the wavelength of the short wavelength band reveal that light focused through a high NA microscope objective scatters with a doughnut shaped profile similar to the profile of the brightest vertical electric dipole (VED) mode. Simulations of scattering of an HO-frequency incident plane wave to the far field reveal that, at this shorter wavelength, however, scattering has lower symmetry than a VED. Finite element calculations also show that the gap field of the higher frequency mode also has lower symmetry than the near field of the VED.

Photonic and Phononic Properties of Engineered Nanostructures II,

edited by Ali Adibi, Shawn-Yu Lin, Axel Scherer, Proc. of SPIE Vol. 8269

82691M - @ 2012 SPIE · CCC code: 0277-786X/12/\$18 - doi: 10.1117/12.907700

Proc. of SPIE Vol. $826982691 \mathrm{M}-1$ 
Further analysis of the character of the high frequency band is performed using a dielectric function with reduced loss which allows resolution of the underlying HO modes. The band is found to be comprised of two modes, one excited only under conditions of p-polarized excitation and another that is excited by either p- or s-polarized light.

\section{METHODS}

BV method: The far-field scattering spectra are calculated by the method developed by Bobbert and Vlieger (BV). This method which includes the multipolar reflection of the particle scattering, extends the Sommerfeld's method calculating the dipole interacting with a planar surface. The computer code based on this BV method is implemented by Germer. For the computational model, a $50 \mathrm{~nm}$ gold particle is placed above a dielectric film with $0.7 \mathrm{~nm}$ thickness and refractive index 1.65. Additional $0.3 \mathrm{~nm}$ spacing is inserted between the particle and film for the convergence purpose. The gold substrate with refractive index from Johnson and Christy's (J-C) experimental data is used below the dielectric film. The scattering intensity is integrated over the light cone spanning $64^{\circ}$ around the $+\mathrm{z}$ axis. This angle is corresponding to maximum collection angle of an objective lens with numerical aperture of 0.9 .

FEM: To calculate the scattered field in the near-field region, the finite element method (FEM) based full wave calculation is performed (COMSOL 3.5a). The excitation field is predefined by calculating the total field in the same model without the nanoparticle. In this case, this total field is obtained by solving a problem of a multilayer reflection system with incident wave at oblique angle. The incident angle is at $70^{\circ}$ from normal in all following FEM calculation. The perfect match layer (PML) is used as boundary condition.

\section{FAR-FIELD CALCULATION}

The previous experimental results indicate that the HO gap modes exist and it has different scattering properties compared to the gap dipole mode. Therefore, in the following section, the simulation based on B-V method and FEM are used to explore the far-field and near-field properties of these gap modes.

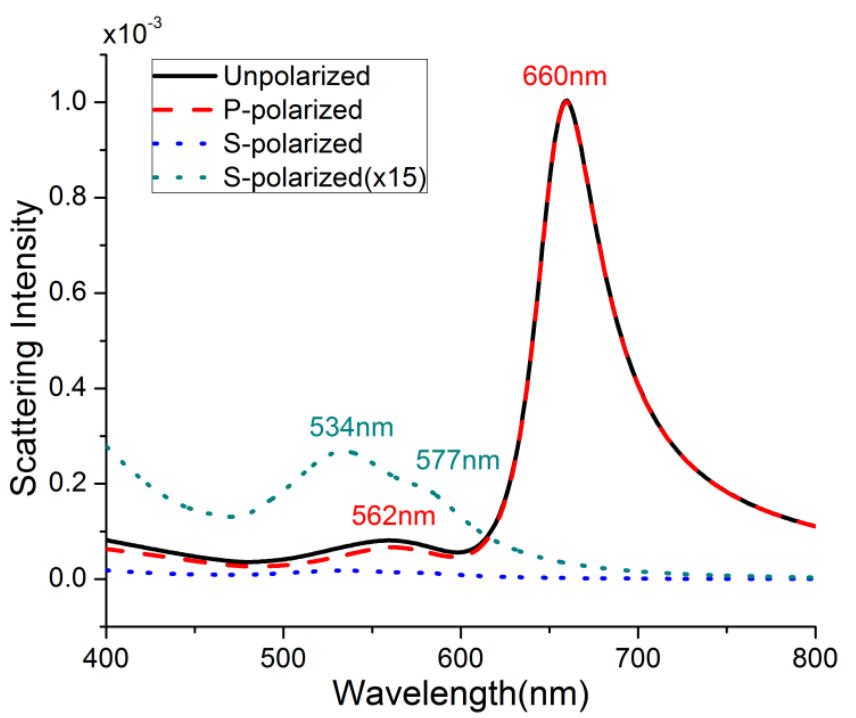

Figure 1 The B-V method simulated scattering spectra excited by different polarization. 

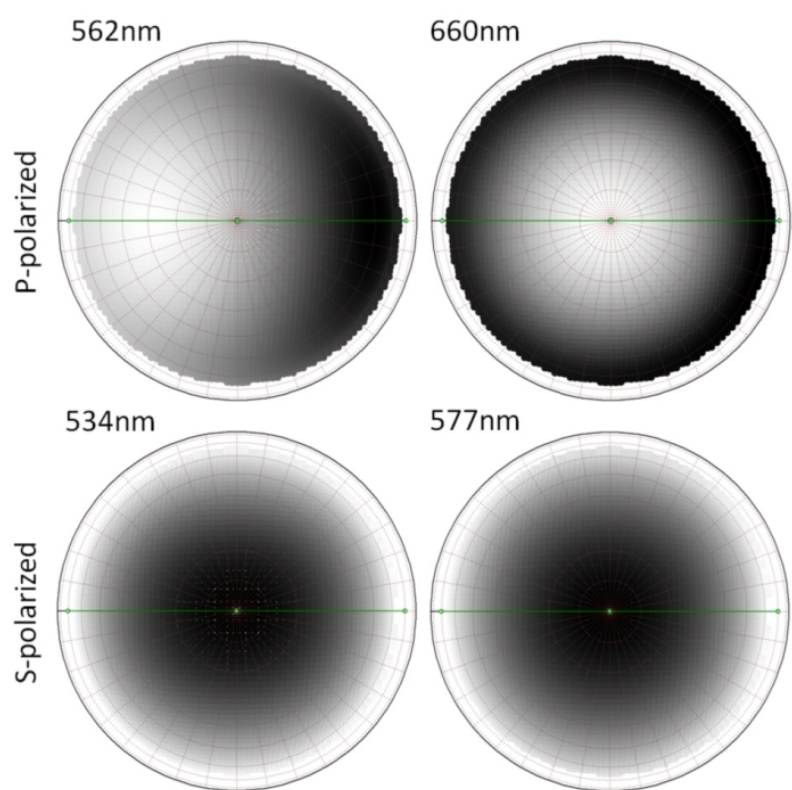

Figure 2 The far-field scattering profiles at the wavelength of spectral features.

The scattering spectra and profile simulated by B-V methods are shown. In Figure 1, for the scattering spectrum of unpolarized excitation, the gap dipole mode and the HO mode are mainly contributed from p-polarized component of excitation. The gap dipole mode is located at $660 \mathrm{~nm}$ and the $\mathrm{HO}$ is at $560 \mathrm{~nm}$. However, for p-polarized excited spectrum, the HO mode is at $562 \mathrm{~nm}$. This slightly peak sift is due to the s-polarized excited spectrum peaked at $534 \mathrm{~nm}$. The s-polarized excited spectrum also has a shoulder peak around $585 \mathrm{~nm}$. This spectrum shape is different from the isolated particle scattering spectrum. This indicates that not only p-polarized excitation but also s-polarized excitation can result in the interaction between the particle and film.

We choose four plasmonic bands: $534 \mathrm{~nm}, 562 \mathrm{~nm}, 577 \mathrm{~nm}$, and $660 \mathrm{~nm}$ to observe the scattering profile, Figure 2 . These wavelengths are chosen from the features of scattering spectra with p-polarized only and s-polarized only excitation. For p-polarized excitation, the gap dipole mode and HO mode have different scattering profiles. The gap dipole shows doughnut shape but $\mathrm{HO}$ mode has asymmetric pattern around z-axis at $562 \mathrm{~nm}$. For s-polarized excitation, the far-field scattering profiles are all symmetric around z-axis and the maximum is in the center.

To identify these gap modes, first we tried to find the most efficient configuration to excite them. Although we already know p-polarized component is dominant, the s-polarization excited spectra are included for comparison, Figure 3 . The incident angle is tuned from $0^{\circ}$ to $89^{\circ}$. The intensity for gap dipole mode reaches the maximum at $75^{\circ}$. For the HO modes, at the small angle $\left(\sim 0^{\circ}\right.$ to $\left.30^{\circ}\right)$, the free-particle-like mode is excited and then red shifted to the higher order plasmonic gap mode. This $\mathrm{HO}$ mode reaches the maximum at $70^{\circ}$. For s-polarized excitation, there are two peaks one is also a free-particle-like mode at $531 \mathrm{~nm}$ and the other is around $585 \mathrm{~nm}$. The intensity of these two modes decreases as the angle of incident increases from normal direction. 


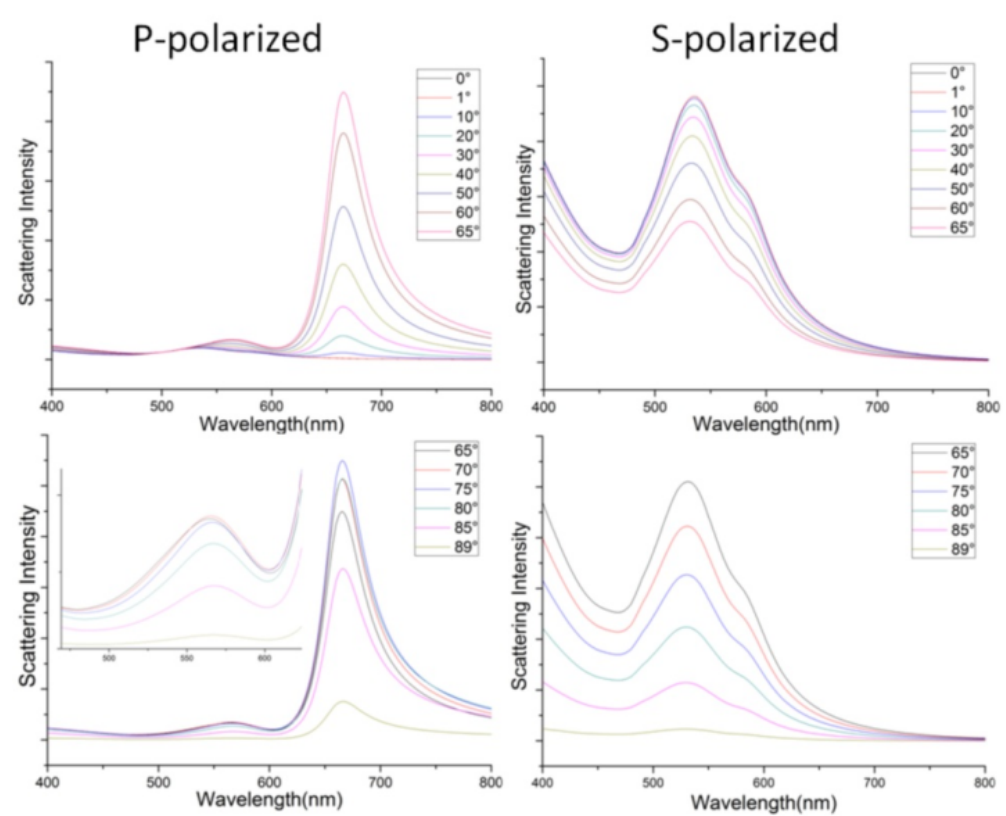

Figure 3 The angle dependence of the plasmonic modes for P-polarized excitation (left) and S-polarized excitation (right).

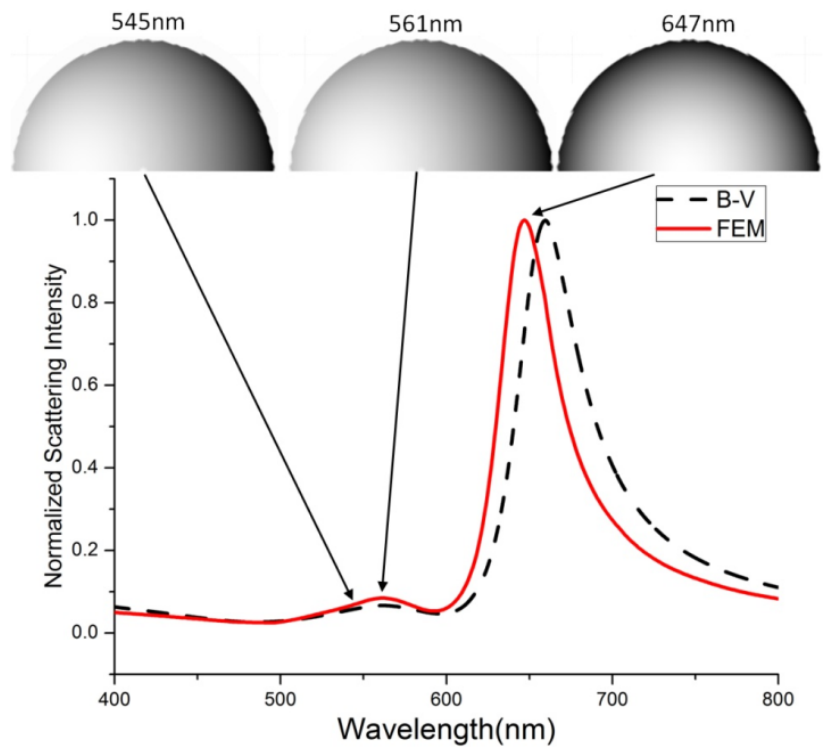

Figure 4 (Down) The scattering spectra simulated by FEM (red) and BV (black dashed) method. (Up) The scattering profile calculated by FEM at the plasmonic bands.

\section{NEAR-FIELD CALCULATION}

FEM is utilized to calculate the scattered field from near-field region to far-field region. The electric field around the particle/film gap describes the plasmonic mode profile. To verify this scattered field calculation, the scattering spectrum calculated by FEM is compared with the spectrum calculated by the B-V method, Figure 4. According to the previously simulation, we know that only p-polarized excitation contributes to these gap modes. Therefore, in the following FEM calculation, only p-polarized excitation is considered. As shown in the Figure 4, the FEM calculated spectrum has similar features to the B-V method calculated spectrum except for the slightly blue shift of the peak wavelength. The gap dipole mode has $13 \mathrm{~nm}$ shift and HO mode has $1 \mathrm{~nm}$ shift. 
In addition to spectral features, the FEM calculated far-field scattering profile is also compared to the B-V calculated profile, Figure 5. For the FEM calculated dipole gap mode at $647 \mathrm{~nm}$, the far field scattered field is consistent to the B-V calculation which has clear doughnut shape, Figure 2. For the HO modes at $561 \mathrm{~nm}$, the scattering also has asymmetrical profile, which has maximum at the opposite side of the incident wave. The profile at $545 \mathrm{~nm}$ is also shown to compare with the profile mode appearing when less lossy dielectric function is used in the following simulations.

The near field distribution on two surfaces is demonstrated, Figure 5. One is on X-Z plane which is the incident plane and the other is on the particle surface facing the film. As shown in Figure 5, for all three wavelengths, Ez is dominant and Ex is roughly one order smaller. For the gap dipole mode at $647 \mathrm{~nm}$, the field is highly concentrated in the dielectric layer, roughly $1 \mathrm{~nm}$ by $1 \mathrm{~nm}$ in this cross section. The enhancement of Ez for gap dipole mode is around 200, which is four times larger than the one for $\mathrm{HO}$ mode at $561 \mathrm{~nm}$. The highest Ez field is located in the air gap between particle and the film instead of dielectric layer for the $\mathrm{HO}$ modes at $561 \mathrm{~nm}$. In addition, the distribution is not symmetric to the Z-axis. For HO modes at $545 \mathrm{~nm}$, the Ez field has concentrated spots with opposite phase located in the dielectric film.

In Figure 5, for the scattered field on the particle surface, the gap dipole mode has one lobe on the surface, which is symmetrical around $\mathrm{z}$ axis. For the HO mode, two lobes with different phase are observed.
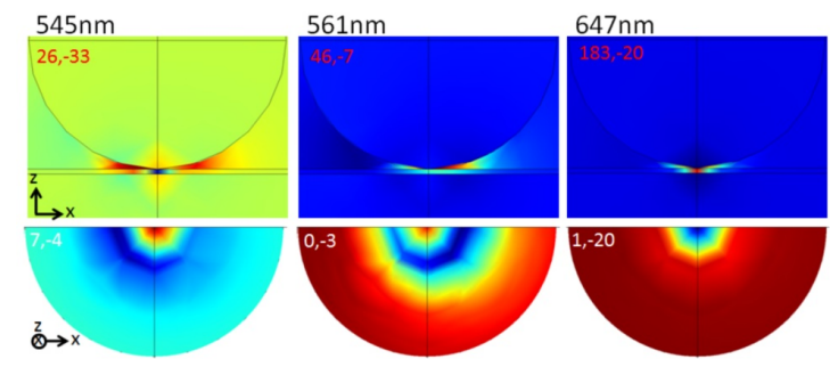

Figure 5 (Up) The near-field distribution of three plasmonic bands on X-Z plane. (Down) The near-field distribution of three plasmonic bands on the particle surface facing the film. The rainbow colorbar is used and the set of number indicates the maximum and minimum value of the field.

\section{FAR-FIELD CALCULATION WITH REMOVAL OF INTERBAND LOSS}

From the previous experimental scattering profiles, calculated spectra, and near-field distribution, it indicates that the HO modes may be contributed from more than one mode. To clarify this, in the following simulations, we replace the J-C dielectric function into Drude model to remove the interband loss. The removal of interband loss can magnify the plasmoic modes, especially for the modes between $500 \mathrm{~nm}$ to $600 \mathrm{~nm}$. The parameters we used for the Drude model are list below: $\varepsilon_{\infty}=9.5, \omega_{\%}=8.9488_{r} \gamma=0.01$.

Both p-polarized and s-polarized can excite these plasmonic modes except that gap dipole mode at $656 \mathrm{~nm}$ and the mode at $542 \mathrm{~nm}$ can only excited by p-polarized light. These two modes have similar doughnut shape profile, Figure 6 . For the mode at $568 \mathrm{~nm}$, which can be both excited by p-polarized and s-polarized light, the mode has maximum along zaxis. However, the p-polarized excited mode at $568 \mathrm{~nm}$ is slightly tilted because of the coupling from the $542 \mathrm{~nm}$ mode. The s-polarized excited $568 \mathrm{~nm}$ mode provide a clean profile of this mode. According to the scattering spectrum, the original HO mode observed from experiments and simulated spectrum may possibly result from band at $542 \mathrm{~nm}$ and 568 $\mathrm{nm}$ in this scattering spectrum calculated based on Drude dielectric function. 

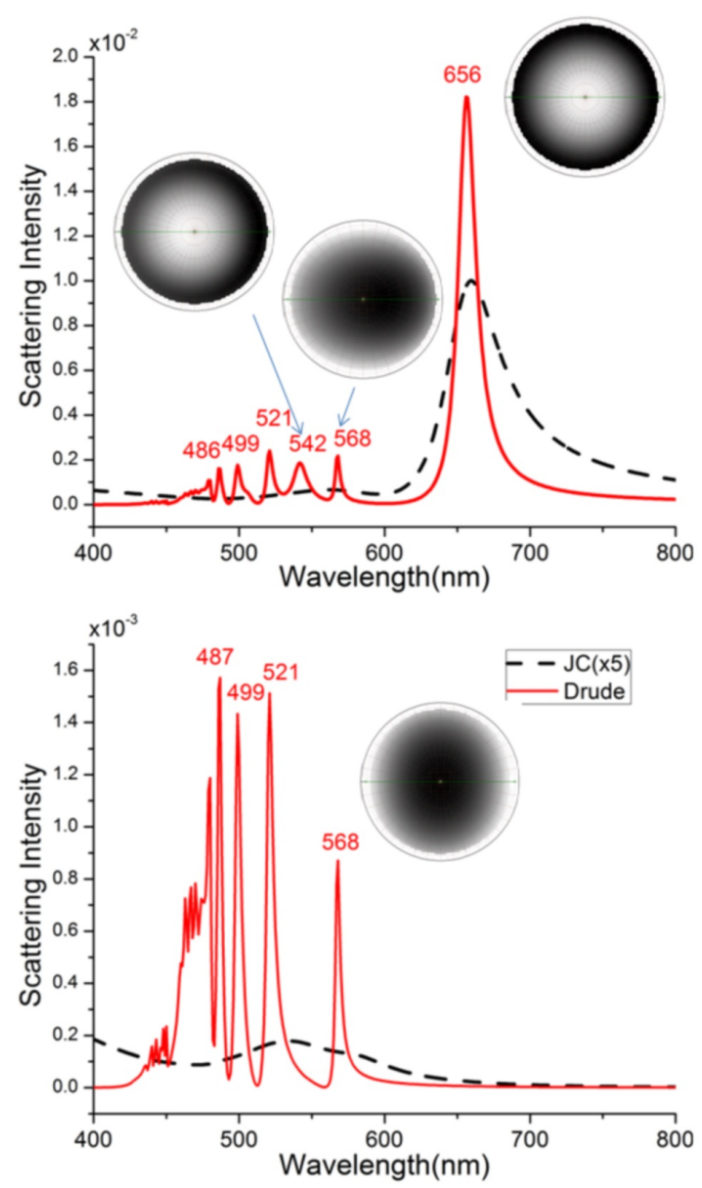

Figure 6 The scattering spectra under JC dielectric function and Drude dielectric function. (Up) The excitation is ppolarized at $70^{\circ}$. (Down) The scattering spectra by JC and Drude dielectric function under s-polarized excitation at $70^{\circ}$. The scattering profiles of three plasmonic bands under $\mathrm{p}$ - and s-polarized excitation.

\section{NEAR-FIELD CALCULATION WITH REMOVAL OF INTERBAND LOSS}

With the same Drude dielectric function, the FEM is used again to calculate the near-field profile of these plasmonic modes. Same as in the previous steps verifying the FEM calculation, in Figure 7, the FEM calculated spectrum is compared with the B-V method calculated spectrum. The number of distinguishable modes is the same but there is blue shift for the modes at $508 \mathrm{~nm}, 557 \mathrm{~nm}$ and $644 \mathrm{~nm}$. In addition, the modes are more distinct in the B-V method calculated spectrum. In Figure 7, it also shows the scattering profile of the plasmonic modes at $545 \mathrm{~nm}, 557 \mathrm{~nm}$ and 644 $\mathrm{nm}$. Same as in the B-V calculated profile in Figure 4, the gap dipole at 644 and HO mode at $545 \mathrm{~nm}$ both have doughnut shape profile. The HO modes at $557 \mathrm{~nm}$ is slightly different from the mode at $568 \mathrm{~nm}$ in Figure 4, which may come from more coupling between to the mode at $545 \mathrm{~nm}$ according to the spectrum in Figure 7.

The near-field profile of gap dipole mode at $644 \mathrm{~nm}$ and HO modes at $557 \mathrm{~nm}$ and $545 \mathrm{~nm}$ is demonstrated in Figure 8 . In general, the model profile of the modes at $644 \mathrm{~nm}$ and $545 \mathrm{~nm}$ are the similar to the corresponding ones by using J-C dielectric function except that the maxima Ez is increase by the factor of 5 due to removal of the interband loss.

In Figure 8, owing to the decoupling from the adjacent mode, for the HO mode at $557 \mathrm{~nm}$, the two lobes of Ez across the z-axis becomes more obvious. This Ez contribution corresponds to the quadrupole distribution of the charge density. 


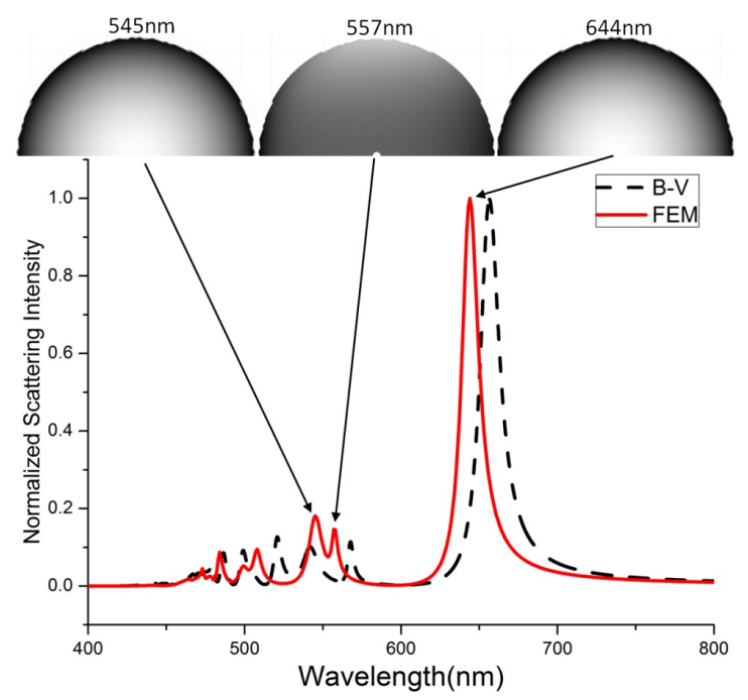

Figure 7 (Down) The scattering spectra calculated by FEM (red) and BV method (black dashed) with Drude dielectric function. (Up) The scattering profile calculated by FEM at the plasmonic bands.
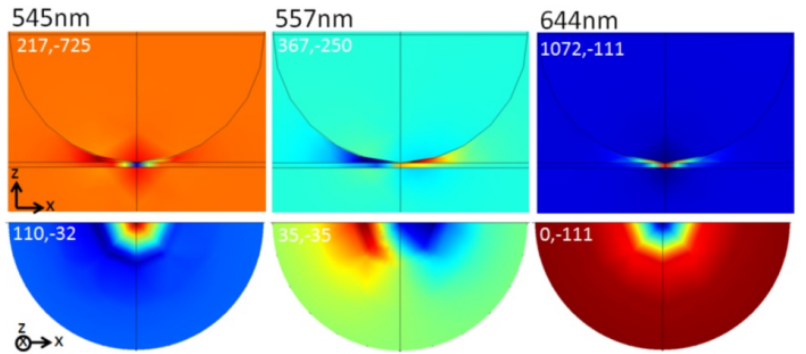

Figure 8 (Up) The near-field distribution of three plasmonic bands on X-Z plane. (Down) The near-field distribution of three plasmonic bands on the particle surface facing the film. The rainbow colorbar is used and the set of number indicates the maximum and minimum value of the field.

\section{CONCLUSION}

In conclusion, the gap dipole mode and HO mode observed in the experiments can be clarified by the spectrum and scattering profile either by BV method or FEM. These two plasmonic modes can be distinguished by far-field scattering spectrum and scattering profile. Under our collection scheme, these modes are mainly excited by the p-polarized light illuminating at $70^{\circ}$ from normal direction. With the help of less lossy dielectric function, the HO modes can be decomposed into the two gap mode at $545 \mathrm{~nm}$ and $557 \mathrm{~nm}$. These two gap modes have different properties. In terms of near field distribution of Ez, the $545 \mathrm{~nm}$ mode is more dipole-like and $557 \mathrm{~nm}$ mode is more quadrupole-like. The damping and then coupling of these two modes may lead to the asymmetrical far-field scattering profile shown in the experiments and simulations.

\section{REFERENCE}

[1] F. Le, N. Z. Lwin, J. M. Steele, M. Käll, N. J. Halas, and P. Nordlander, "Plasmons in the metallic nanoparticlefilm system as a tunable impurity problem.," Nano Lett 5, 2009-2013 (2005) [doi:10.1021/n10515100].

[2] S.-Y. Chen, J. J. Mock, R. T. Hill, A. Chilkoti, D. R. Smith, and A. A. Lazarides, "Gold nanoparticles on polarizable surfaces as Raman scattering antennas.," ACS Nano 4, 6535-6546 (2010) [doi:10.1021/nn101644s]. 